\title{
SMASIS2016-9137
}

\section{OPERATIONAL SPACE CONTROL OF A LIGHTWEIGHT ROBOTIC ARM ACTUATED BY SHAPE MEMORY ALLOY (SMA) WIRES}

\author{
Serket Quintanar-Guzmán* \\ Interdisciplinary Centre for Security, Reliability and Trust \\ University of Luxembourg \\ Luxembourg, 1359 \\ Luxembourg \\ Email: serket.quintanar@uni.lu
}

\author{
Somasundar Kannan, \\ Miguel A. Olivares-Mendez, \\ Holger Voos \\ Interdisciplinary Centre for Security, Reliability and Trust \\ University of Luxembourg \\ Luxembourg, 1359 \\ Luxembourg
}

\begin{abstract}
This paper presents the design and control of a two link lightweight robotic arm using a couple of antagonistic Shape Memory Alloy (SMA) wires as actuators. A nonlinear robust control law for accurate positioning of the end effector of the twolink SMA based robotic arm is developed to handle the hysteresis behavior present in the system. The model presented consists of two subsystems: firstly the SMA wires model and secondly the dynamics of the robotic arm itself. The control objective is to position the robotic arm's end effector in a given operational plane position. For this regulation problem a sliding mode control law is applied to the hysteretic system. Finally a Lyapunov analysis is applied to the closed-loop system demonstrating the stability of the system under given conditions. The simulation results demonstrate the accurate and fast response of the control law for position regulation. In addition, the stability of the closed-loop system can be corroborated.
\end{abstract}

\section{INTRODUCTION}

Shape Memory Alloys (SMA) consist of a group of metallic materials which can return to their initial shape and size after been deformed, when subjected to the appropriate thermal procedure. This phenomenon is known as Shape Memory Effect

\footnotetext{
*Address all correspondence to this author.
}

(SME). The Niquel-Titanium SMA wires (known as NiTi wires) are one of the most common types of SMA.

The SME occurs due to an inner transformation of the material's crystalline structure. This transformation happens between two phases called martensite and austenite. When the SMA wire is at lower temperature its structure shifts to the martensite phase which is a relatively soft and malleable phase, during wich the wire can be easily deformed. When heated over the transformation temperature the SMA wire transforms into the austenite phase, a hard phase, recovering its initial form and size.

The high force to mass ratio, small size, noiseless operation, and bio-compatibility have made NiTi wires a great alternative to conventional hydraulic, electric and pneumatic actuators. However, they still represent a huge challenge for accurate implementation, due to its slow and highly non-linear response.

Nowadays SMA wires are being used in a wide range of applications, from art-craft designs [1], to medical implements like intra-arterial supports [2] or dental applications [3], construction vibrations damper [4], micro-robotics [5, 6], specific purpose actuators as camera lense focus actuator [7], car mirror actuators [8] or SMA based motors [9], general purpose actuators [10-15] and robotic manipulators as arms, hands or robotic fingers [16-18]. Most of the mentioned applications are microscale or required complicated mechanical systems to be implemented. This paper presents a new design for a SMA wire ac-
Copyright (C) 2016 by ASME 
tuated robotic arm. Our proposal keeps the simplicity of the mechanics and therefore achieves a light-weight actuator. This light-weight characteristic is critical in applications like robotic manipulator for unmanned aerial vehicles (UAVs), wich will be the main purpose of this arm. It is a great challenge to make an optimal use of available payload of an aerial vehicle such as a quadcopter. We propose a lightweight design, which enables the arm to be implemented without significantly decreasing the quadcopter's available payload.

The paper is organized as follows: Section describes the mechanical design and mathematical model of the proposed robotic arm. Section presents the non-linear applied control law as well as the operational space control law, followed by the stability analysis developed in section. The results are discussed in section. Finally the conclusion are presented in Section .

\section{SMA ACTUATED ROBOTIC ARM}

This section presents the mechanical design of the SMA actuated robotic arm as well as the mathematical model of the overall system. The mathematical model of the SMA wires, as well as its kinematic and dynamic relation with the mechanical model is explained in detail. Finally the modeling approach for the mechanical system based on the CAD (Computer Aided Design) model is illustrated.

\section{Mechanical Design}

In this section we present the mechanical design of a lightweight SMA actuated robot arm. The optimal use of available payload of an aerial vehicle is critical for aerial manipulators design, with this in mind we propose a single Degree of Freedom (DOF) actuator activated by a couple of antagonistic SMA wires. Figure 1 shows a CAD model of the robot arm design. The design is based on an existing joint proposed in [12]. This joint consists of two couplers joined by a torsion spring. Each SMA wire is attached in one end to its respective coupler allowing to control independently the angular position of each coupler. The SMA wire attached to coupler 2 allows to increase or decrease the stiffness of the joint as required by adjusting the torque applied by the torsion spring, while SMA 1 affects directly the angular position of the end effector $\left(\theta_{1}\right)$ by controlling the angular position of coupler 1 (see Fig. 1).

The given robotic arm with $1 \mathrm{DOF}$, is activated by two $37 \mathrm{~cm}$ long NiTi wires. It has two custom made carbon fiber links (150 $\mathrm{mm}$ and $100 \mathrm{~mm}$ respectively) and a range of movement along the vertical plane $X-Z$ up to 85 degrees with two $7.5 \mathrm{~mm}$ radius couplers. It has a total weight of $48 \mathrm{~g}$, which is about $25 \%$ of the weight respect to other light-weight designs found in the literature as the one presented in [19]. The winding wheels enable the use of longer SMA wires to increase the movement range without increasing the dimension of the links.

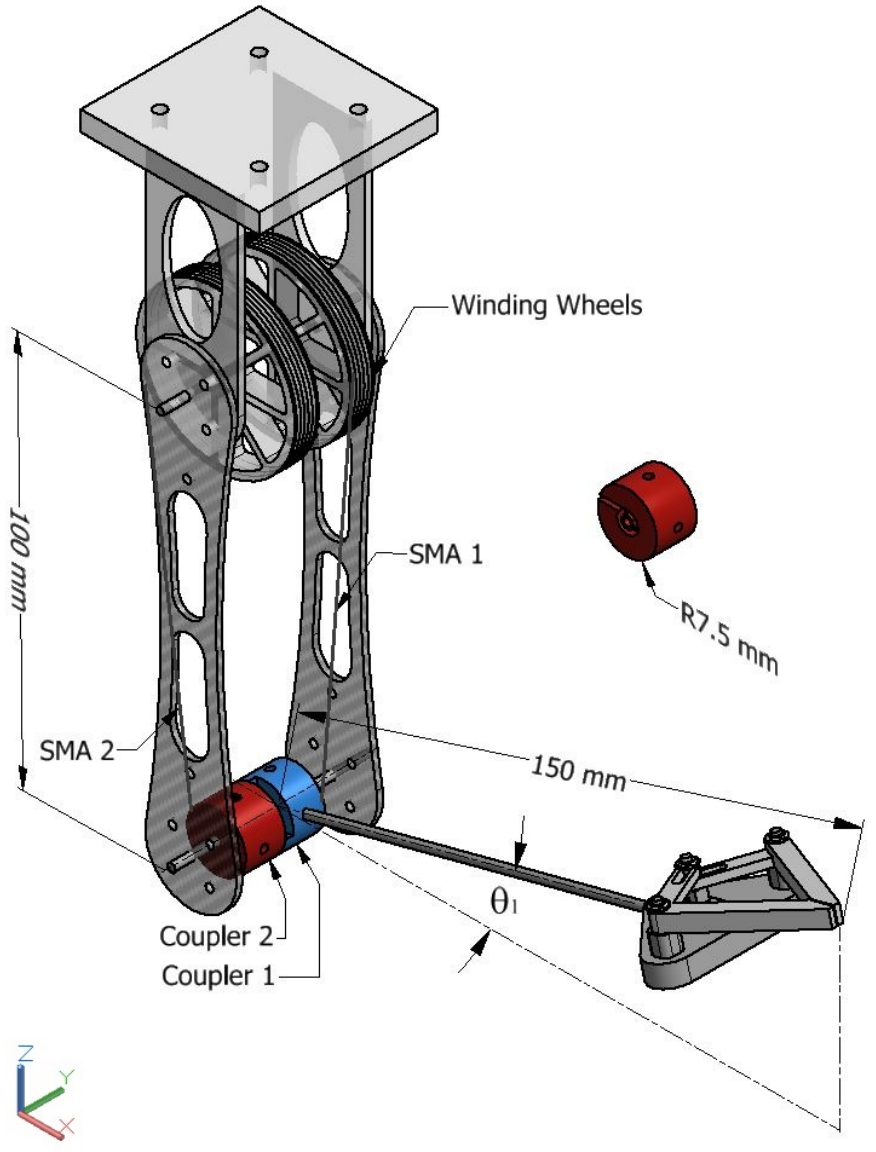

FIGURE 1: PROPOSED SMA WIRE ACTUATED ROBOTIC ARM CAD MODEL

\section{System Modeling}

The overall system model is composed of several subsystems. Figure 2 shows the block diagram of the robotic arm's mathematical model. This model is divided in two main subsystems: 1) Operational Space Control and 2) Actuator and inner control. The latter, in turn, is formed by three other subsystems: Inner control law, SMA Model and Kinematic and Dynamic model. As shown in Fig. 2, the Actuator and Inner control subsystem has several cross-coupling effects and is considered as a MIMO (multiple-input and multiple-output) system with two inputs $\left(V_{1}, V_{2}\right)$ and two outputs $\left(\theta_{1}, \theta_{2}\right)$.

The Actuator and Inner control contains the SMA wire subsystem, and its schematic model is illustrated in Fig.3. This SMA wire subsystem is described by a mathematical model of the NiTi wires which was proposed by the authors in [11]. This is also divided in three subsystems representing the thermal dynamics, the heat transformation and the constitutive model. In this figure the interaction between the variables of each subsystem of the SMA 


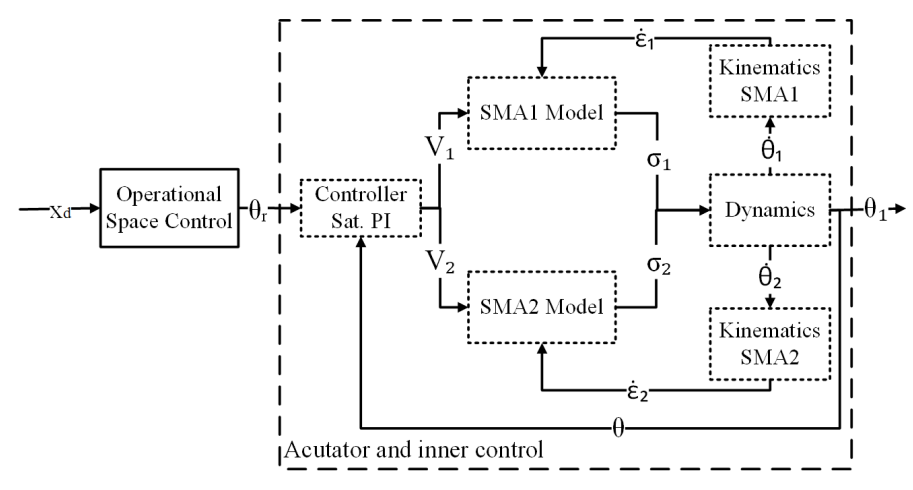

FIGURE 2: BLOCK DIAGRAM OF THE SMA ACTUATED ROBOTIC ARM

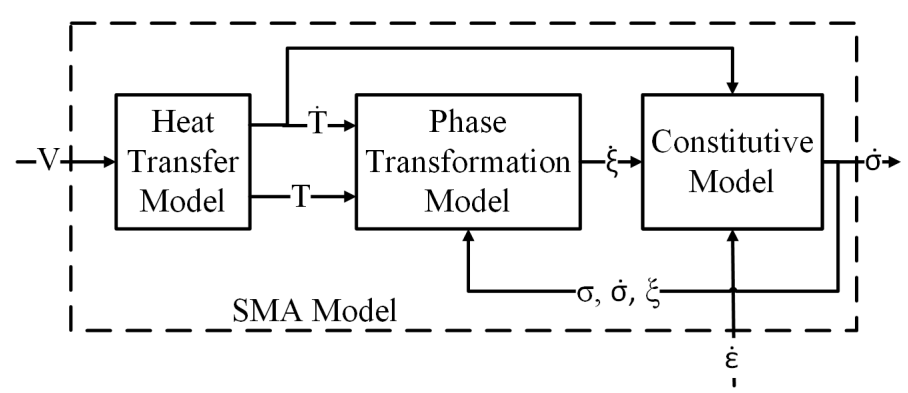

FIGURE 3: SMA WIRE MATHEMATICAL MODEL BLOCK DIAGRAM

wire model is shown. On the other hand, the dynamics of the arm is directly derived from a Computer Aided Design (CAD) model. Each of the mentioned subsystems will be explained in more detail in the following subsections.

Heat Transfer Model. The heat transfer block consists of the electrical heating (Joule effect) and the natural convection model described by the following equation [11]:

$$
m_{w} c_{p} \frac{d T}{d t}=\frac{V^{2}}{R}-h A_{w}\left(T-T_{a m b}\right)
$$

where $V$ is the voltage, $R$ is the electric resistance per unit length, $c_{p}$ is the specific heat, $m_{w}$ is the mass per unit length, $A_{w}$ is the wire surface area, $T_{a m b}$ the ambient temperature and $T$ the SMA wire temperature. Here $h$ is approximated by a second order polynomial of the temperature:

$$
h=h_{0}+h_{2} T^{2}
$$

SMA Wire Phase Transformation Model. As shown in Fig. 3, the block containing the phase transformation model computes the martensite fraction $(\xi)$. The phase transformation of the SMA wire depends directly on the direction of the time derivative of the temperature. Therefore, due to hysteresis behavior two equations are needed to fully describe the phase transformation phenomenon. The phase transformation from martensite to austenite while heating is given by

$$
\xi=\frac{\xi_{M}}{2}\left\{\cos \left[a_{A}\left(T-A_{S}\right)+b_{A} \sigma\right]+1\right\}
$$

for $A_{s}+\frac{\sigma}{C_{A}} \leq T \leq A_{f}+\frac{\sigma}{C_{A}}$

Inversely, the transformation from austenite to martensite, during cooling is described by the following dynamic equation:

$$
\xi=\frac{1-\xi_{A}}{2} \cos \left[a_{M}\left(T-M_{F}\right)+b_{M} \sigma\right]+\frac{1+\xi_{A}}{2}
$$

for $M_{s}+\frac{\sigma}{C_{M}} \leq T \leq M_{f}+\frac{\sigma}{C_{M}}$

where $M_{s}, M_{f}, A_{s}, A_{f}$ are the start and end transformation temperatures for martensite and austenite transformation respectively. And $a_{A}=\frac{\pi}{\left(A_{f}-A_{s}\right)}, a_{M}=\frac{\pi}{\left(M_{S}-M_{f}\right)}, b_{A}=-\frac{a_{A}}{C_{A}}, b_{M}=-\frac{a_{M}}{C_{M}}$, $C_{A}$ and $C_{M}$ are curve fitting parameters.

Wire Constitutive Model. The wire constitutive model describes the relation between stress $\sigma$, strain $\varepsilon$, temperature $T$ and martensite fraction $\xi$. The general form, firstly proposed in [20] and then modified in [11], is written as

$$
\dot{\sigma}=E \dot{\varepsilon}+\Omega \dot{\xi}+\Theta \dot{T}
$$

The authors in [11] propose a constant value for the Young's modulus $E$ as an average of the Young's modulus of each phase austenite $\left(E_{A}\right)$ and martensite $\left(E_{M}\right)$. However, since the actuator presented here uses antagonistic SMA wires, the Young's modulus depends of the martensite fraction as follows:

$$
E=\xi E_{M}+(1-\xi) E_{A}
$$

here $\Omega$ and $\Theta$ represent the phase transformation constant and thermal expansion coefficient, respectively. Where

$$
\Omega=-E \varepsilon_{0}
$$

and $\varepsilon_{0}$ is the initial strain. 
Kinematic Model and Dynamics. In this section the model of the mechanical design and its relation with the rest of the system is explained.

Kinematic Model. The kinematic model relates the SMA wire model with the mechanics of the robotic arm itself. The strain ratio of the SMA wire and angular velocity of the arm are related kinematically as:

$$
\dot{\varepsilon}_{i}=-\frac{r_{i} \dot{\theta}_{i}}{l_{0 i}}
$$

where $r_{i}$ is the respective coupler radius, $l_{0}$ the initial length of each wire and $\dot{\theta}_{i}$ the angular velocity of each coupler. Equation (8) shows that the angular position of each coupler with respect to the $X$-axis $\left(\theta_{i}\right)$ is inversely proportional to the strain of the wire $\left(\varepsilon_{i}\right)$.

Dynamics. The dynamics describe the relation between coupler mechanism, torsion spring and forces applied by the SMA wires, as well as the effects of the load and grip at the end of the second link. The general dynamic model of the mechanical system is mathematically described as:

$$
J \ddot{\theta}=\left[\begin{array}{c}
\tau_{w 1}(\sigma)-\tau_{s}(\theta)-\tau_{g}(\theta)-\tau_{\text {load }}(\theta)-b_{1} \dot{\theta}_{1} \\
-\tau_{w 2}(\sigma)+\tau_{s}(\theta)-b_{2} \dot{\theta}_{2}
\end{array}\right]
$$

where $b$ is the friction of each coupler and $\tau$ is the torque applied over the mechanical system by the SMA wires (w), the torsion spring (s), the weight of the gripper ( $\mathrm{g}$ ) and the load. This model was developed in two parts: Firstly the coupler mechanism, gripper, load and link dynamics and secondly the mathematical model of $\tau_{w 1}$ and $\tau_{s}$. The dynamic behavior of the couplers, gripper, load and links was directly obtained from the CAD design shown in Fig. 1 developed in Autodesk/Inventor environment.

This model does not only include the exact geometry of each piece but masses, inertias and centers of mass necessary for dynamic analysis. The CAD model is imported via the SimMechanics toolbox in order to obtain a continuous dynamic MATLAB/Simulink model of the mechanical system. On the other hand, the torsions spring and SMA wires torques were obtained from basic physical laws, from which it can be deduced that the SMA wire's force $\left(F_{w}\right)$ is inversely proportional to the stress $(\sigma)$ which can be calculated by integration of Eq. (5):

$$
\tau_{w i}=F_{w i} r_{i}=A \sigma_{i} r_{i}
$$

where $r$ is the coupler radius and $A$ the transversal area of the wire. The torsion spring torque $\tau_{s}$ is calculated as:

$$
\tau_{s}=k_{s}\left(\theta_{1}-\theta_{2}\right)+b_{s}\left(\dot{\theta}_{1}-\dot{\theta}_{2}\right)
$$

where $k_{s}$ is the spring constant and $b_{s}$ is the spring's friction factor, $\theta_{1}$ and $\theta_{2}$ are the angular position of each coupler with respect to $X$-axis.

\section{CONTROL LAW}

The control law presented here is divided into two separate controllers. First is the Inner control law, which works in the Joint Space, which regulates the output angle of the actuator $\theta_{1}$. Second there is the Operational Space Control, which allows to control the end effector poition in Cartesian coordinate system. These two control laws are explained in further detail in the following subsections.

\section{Inner Control Law}

The inner control law regulates the rotational movement of the end effector. The controlled variable is the angular position of coupler $1\left(\theta_{1}\right)$ (see Fig. 1). For the inner control a Variable Structure Control (VSC) is applied, which utilizes a switching control law to force the plant's state trajectory onto a user-specified surface in the state space and maintain it on that surface. Depending if the state is over or under the surface, different control structures are applied. This type of control is considered to be robust against parameter uncertainties, model nonlinearities and external disturbances.

The basic control law is given by

$$
s_{i}=c_{i}+c_{p i} e+c_{I i} \int e d t
$$

where $c_{p i}$ and $c_{I i}$ represent the proportional and integral gains respectively, $c_{i}$ is a small voltage to keep the wires in tension when the position error is zero. The angular position error $e$ is defined as:

$$
e=\theta_{1}-\theta_{r}
$$

The control voltage is defined as

$$
v_{i}=\left\{\begin{array}{l}
V_{i H}, s_{i} \geq \phi_{i} \\
s_{i}, 0 \leq s_{i}<\phi_{i} \\
0, s_{i}<0
\end{array}\right.
$$

where $\phi_{i}$ is the boundary layer and $V_{i H}$ is the maximum voltage. 


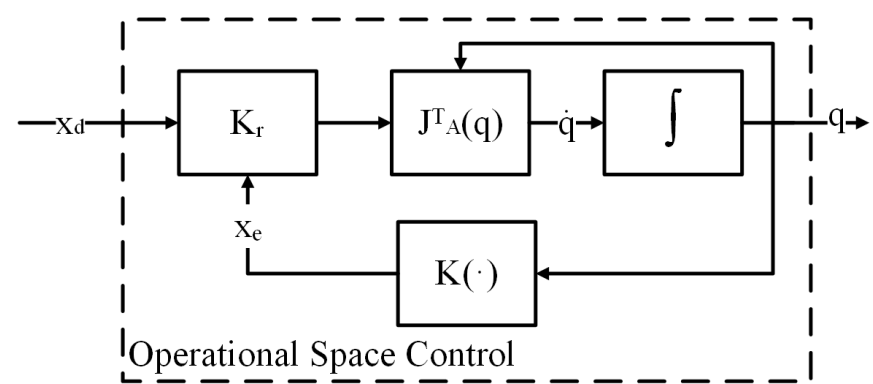

FIGURE 4: OPERATIONAL SPACE CONTROL BLOCK DIAGRAM

\section{Operational Space Control}

A second controller is designed in order to work in an Operational Space, as shown in Fig. 2. This second control has as input the references in a Cartesian coordinate system and gives as output the reference in joint space for the inner control. The schematic diagram of the operational space control is illustrated in Figure 4. This is described by the following equation:

$$
\dot{q}=J_{A}^{T}(q) K_{r} e_{o s}
$$

where $x_{d}$ is the set of Cartesian coordinates for the end effector's desired position, $J_{A}$ is the analytical Jacobian of the robotic arm, $K_{r} \in \mathbb{R}^{n}$ such is a symmetric gain matrix and $e_{o s}$ is the operational space error defined as

$$
e_{o s}=x_{d}-x_{e}
$$

This control represents a simple proportional control which takes into account the direct and inverse dynamics of the one DOF robot arm. Equation (17) shows the analytical Jacobian of the robotic system and Eqn. (18) shows the direct kinematics.

$$
\begin{gathered}
J_{A}(q)=\frac{\partial K(\cdot)}{\partial q}=\left[\begin{array}{c}
-a_{1} \sin \left(q_{1}\right) \\
-a_{1} \cos \left(q_{1}\right)
\end{array}\right] \\
K(\cdot)=\left[\begin{array}{c}
a_{1} \cos \left(q_{1}\right) \\
a_{1} \sin \left(q_{1}\right)-h
\end{array}\right]
\end{gathered}
$$

\section{STABILITY ANALYSIS}

Consider the nonlinear system as follows:

$$
\dot{x}=\left[\begin{array}{c}
\frac{\frac{V_{1}^{2}}{R}-h_{1} A_{w}\left(x_{1}-T_{\text {amb }}\right)}{m_{w} c_{p}} \\
\frac{\frac{V_{2}^{2}}{R}-h_{2} A_{w}\left(x_{2}-T_{\text {amb }}\right)}{m_{w} c_{p}} \\
x_{5} \\
x_{6} \\
\frac{F_{1} r_{1}-b_{1} x_{5}-\tau_{r}-m_{\text {load }} g r_{\text {load }} \cos \left(x_{3}\right)}{J_{1}} \\
\frac{\tau_{r}-F_{2} r_{2}-b_{2} x_{6}}{J_{2}}
\end{array}\right]
$$

Where:

$x_{1}$ - Temperature of SMA wire $1\left(T_{1}\right)$

$x_{2}$ - Temperature of SMA wire $2\left(T_{2}\right)$

$x_{3}$ - Angular position of coupler $1\left(\theta_{1}\right)$

$x_{4}$ - Angular position of coupler $2\left(\theta_{2}\right)$

$x_{5}$ - Angular velocity of coupler $1\left(\dot{\theta}_{1}\right)$

$x_{6}$ - Angular velocity of coupler $2\left(\dot{\theta}_{2}\right)$

Applying a change of variable, the regulation error (position error defined previously in Eqn. (13)) is rewritten as:

$$
e=x_{3}-x_{d}
$$

Let $\bar{x}=\left[\begin{array}{llllll}x_{1} & x_{2} & e & x_{4} & x_{5} & x_{6}\end{array}\right]^{T}$ be the new state vector.

For the system derived from Eqn.(19) and Eqn.(20), a Lyapunov function candidate is proposed as follow:

$$
V(\bar{x})=P_{1} x_{1}+P_{2} x_{2}+P_{3} e^{2}+P_{4} x_{4}^{2}+P_{5}\left(x_{5}+x_{6}\right)^{2}
$$

where $P_{i} \in \mathbb{R}>0, i=1,2,3,4,5$. Assuming $T_{a m b}>0$ for $x_{i} \geq$ $T_{a m b}$ where $i=1,2$ then $V(\bar{x})$ can be considered to be positivedefinitive.

$$
\begin{aligned}
& \text { If } \bar{x}(0)=\left[\begin{array}{llllll}
T_{a m b} & T_{a m b} & 0 & 0 & 0 & 0
\end{array}\right] \text { then } \\
& V(\bar{x}(0))=T_{a m b}\left(P_{1}+P_{2}\right)=x_{e}
\end{aligned}
$$

where $x_{e}$ is the equilibrium point at $t=0$.

The time derivative of the candidate Lyapunov function is:

$\dot{V}(\bar{x})=P_{1} \dot{x}_{1}+P_{2} \dot{x}_{2}+P_{3} e \dot{x}_{3}+P_{4} x_{4} \dot{x}_{5}+P_{5}\left[x_{5} \dot{x}_{5}+\dot{x}_{5} x_{6}+x_{5} \dot{x}_{6}+x_{6} \dot{x}_{6}\right]$

With this result, the function candidate derivative is proven to be negative definite if the conditions set shown in Eqn.(24), Eqn.(25) and Eqn.(26) are fulfilled.

$$
P_{1} \geq \frac{P_{5}\left(x_{5 \max }+x_{6 \max }\right) F_{1 \text { max }} r_{1} m_{w} C_{p}}{J_{1} A_{w} h_{1}\left(x_{1 \max }-T_{a \operatorname{mb}}\right)}
$$




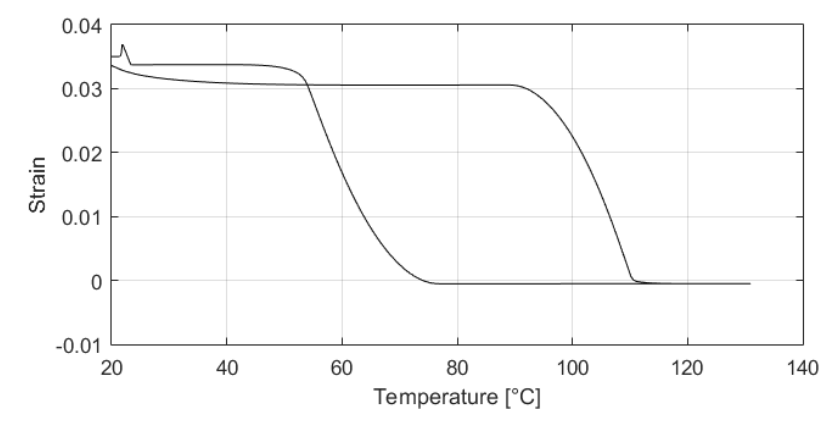

FIGURE 5: OPEN-LOOP SYSTEM HYSTERESIS CURVE STRAIN - TEMPERATURE

$$
\begin{gathered}
P_{2} \geq \frac{P_{5}\left(x_{5 \max }+x_{6 \max }\right) F_{2 \max } r_{2} m_{w} C_{p}}{J_{2} A_{w} h_{2}\left(x_{2 \max }-T_{a m b}\right)} \\
P_{4} \leq \frac{P_{5} b\left(x_{5 \min }+x_{6 \min }\right)^{2}+P_{5}\left(x_{5 \min }+x_{6 \min }\right) k_{1}}{J_{1} x_{4 \min } x_{6 \min }}-\frac{P_{1} V_{H 1}^{2}+P_{2} V_{H 2}^{2}}{m_{w} C_{p} R x_{4 \min } x_{6 \min }}
\end{gathered}
$$

where $x_{\text {imin }} \leq x_{i} \leq x_{\text {imax }}$. These conditions are achievable as long as the following condition is fulfilled

$V_{i H}<\sqrt{\frac{P_{5}\left(x_{5 \min }+x_{6 \min }\right)\left[b\left(x_{5 \min }+x_{6 \min }\right)+m_{\text {load }} g r_{\text {load }}\right]\left(m_{w} c_{p} R\right)}{2 J_{1} P_{1}}}$

The closed-loop system of the plant and inner control is then proven to be asymptotically stable [21]. In our case, for the operational space control system, it is proved that if $K_{r}$ is a positive definite matrix the system is asymptotically stable [22]. Which concludes the proof that overall system is asymptotically stable.

\section{SIMULATION RESULTS}

The SMA actuated robotic arm open and closed-loop performance process was evaluated through simulations using Simulink/MATLAB.

The nonlinear hysteretic characteristics of the system is first evaluated by open loop simulation. This test was performed by applying to the SMA 1 the maximum voltage $\left(V_{1 H}\right)$ while the SMA 2 has no voltage applied and then vice versa. The results of this simulation are shown in Fig. 5. The hysteresis loop generated by this test is the major hysteresis loop. Which is considered a double loop hysteresis and it is generated by the antagonistic SMA wires.

In order to carry out a closed-loop analysis a position regulation was performed. The step response was tested with a series
TABLE 1: PARAMETERS OF THE SMA WIRE AN THE COMPLIANT ACTUATOR $[11,12,23,24]$

\begin{tabular}{cllc}
\hline Parameter & Value & Parameter & Value \\
\hline$E_{M}$ & $28 \mathrm{GPa}$ & $C_{A}$ & $10 \mathrm{Mpa} /{ }^{\circ} \mathrm{K}$ \\
$E_{A}$ & $75 \mathrm{GPa}$ & $C_{M}$ & $10 \mathrm{Mpa} /{ }^{\circ} \mathrm{K}$ \\
$A_{s}$ & $88{ }^{\circ} \mathrm{C}$ & $T_{a m b}$ & $25{ }^{\circ} \mathrm{C}$ \\
$A_{f}$ & $98{ }^{\circ} \mathrm{C}$ & $A$ & $4.9 \times 10^{-8} \mathrm{~m}^{2}$ \\
$M_{s}$ & $72{ }^{\circ} \mathrm{C}$ & $A_{w}$ & $290.45 \times 10^{-6} \mathrm{~m}^{2}$ \\
$M_{f}$ & $62{ }^{\circ} \mathrm{C}$ & $c_{p}$ & $320 \mathrm{~J} / \mathrm{Kg}^{\circ} \mathrm{C}$ \\
$m_{w}$ & $6.8 \times 10^{-4} \mathrm{~kg} / \mathrm{m}$ & $\varepsilon_{L}$ & $2.3 \%$ \\
$R$ & $20 \Omega / \mathrm{m}$ & $h_{0}$ & 20 \\
$l_{0}$ & $0.37 \mathrm{~m}$ & $h_{2}$ & 0.001 \\
$b_{s}$ & 0.5 & $b_{1}, b_{2}$ & 0.1 \\
$k_{s}$ & $0.0018 \mathrm{Nm} / 1^{\circ}$ & $\Theta$ & -0.055 \\
\hline
\end{tabular}

of increased and decreased steps every 40 s with an amplitude computed by Eqn. (28) as shown in Fig. 6 (solid line)

$$
x_{d}=\left[\begin{array}{c}
a_{1} \cos (N(i) \pi / 180) \\
a_{1} \sin (N(i) \pi / 180)-h
\end{array}\right]
$$

where $N=[10,20,30,40,50,40,30], a_{1}$ is the longitude of the second link $(150 \mathrm{~mm})$ and $h$ is the longitude of the first link (100 $\mathrm{mm})$ plus the base high $(50 \mathrm{~mm})$. The origin of the system is set at the center of the upper face of the robotic arm's base. The parameters of the system used for simulation are listed in Tab. 1 and were taken from manufacturer in $[23,24]$ and $[11,12]$. The Operational Space Control gain $\left(K_{r}\right)$ was set at 300 in order to achieve a fast response through this controller. The inner control was set as follows: The boundary layers $\phi_{1}=10$ degrees, $\phi_{2}=7$ degrees, and limit voltage $V_{1 H}=6.5 \mathrm{~V}, V_{2 H}=6.5 \mathrm{~V}$. The results of this simulation are shown in Fig. 6.

In Fig. 6 it can be seen that there is an overshoot. This is attributed to the low friction factor of the system. The maximum overshoot is $13.2 \%$ for positive steps and $6.5 \%$ for negative steps in $X$-axis (see Fig. 6a), while for $Z$-axis (see Fig. 6 b) is $21 \%$ and $12.72 \%$ respectively. In addition, the maximum steady state error in $X$-axis is $0.7 \%$ and $1.6 \%$ for $Z$-axis (see Fig. 7), with an average settling time of 6 seconds. These maximum overshoot and maximum steady state error occur at the furthest position from the initial position, this condition is attributed to the transforma- 


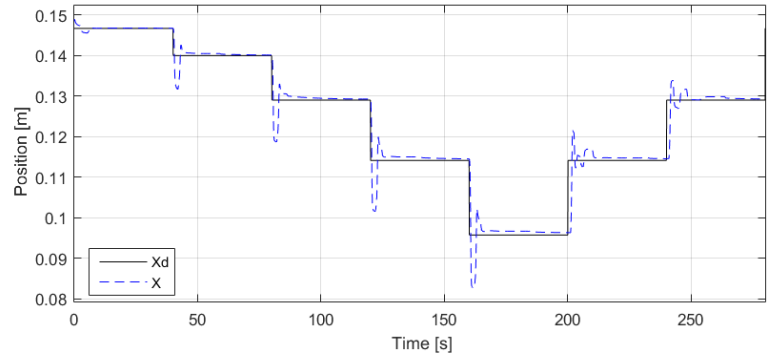

(a) STEP RESPONSE IN X AXIS

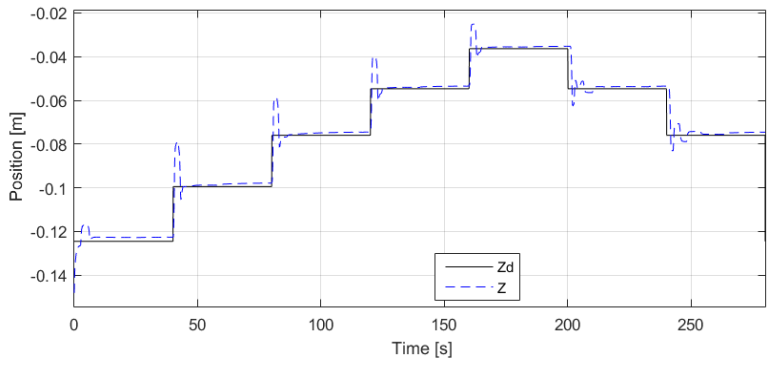

(b) STEP RESPONSE IN Z AXIS

FIGURE 6: STEP RESPONSE IN CARTESIAN COORDINATE FRAME

tion to operational space due to differences in reference frames in the mechanical CAD model. Figure 8 illustrates the inputs of the system during the closed-loop test. The inputs are given in Volts and they are limited to avoid thermal damage to the SMA wires, which can destroy its memory effect. Both SMA wires have the same high voltage limit, however the boundary layer for each wire is set at different levels as mentioned before. The higher limit for $\phi_{1}$ is fixed in order to achieve a faster response from the control law. SMA 2 adjusts the stiffness of the joint. This means that the SMA 2 does not actuate directly over the end effector, thus its rate of response is not as critical as SMA 1.

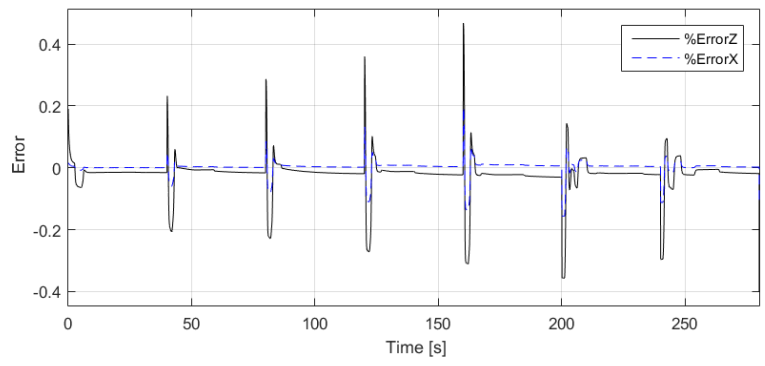

FIGURE 7: STEP RESPONSE ERROR IN X AND Z

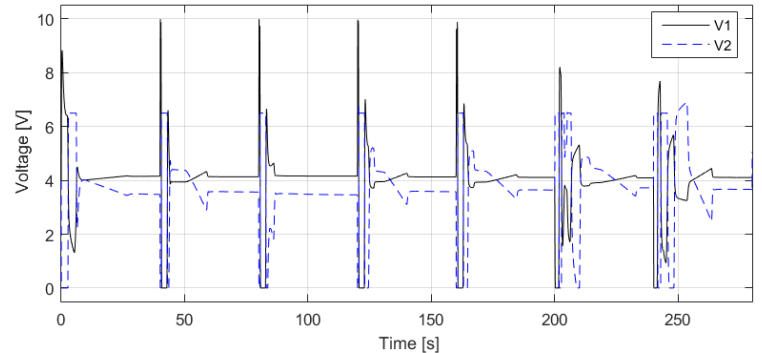

FIGURE 8: VOLTAGE INPUT

\section{CONCLUSION}

We have presented a SMA wire actuated light-weight robotic arm, which is intended to be an alternative for flying manipulators. This arm is actuated by a couple of antagonistic SMA wires. It has a total weight of $48 \mathrm{~g}$ and a range of movement up to 85 degrees on the $X-Z$ plane. Operational Space Control was applied for position regulation, while a sliding mode control was applied as inner control.

Open and closed-loop test were developed. The closed-loop simulation results showed the capability of the variable structure control to deal with the double loop hysteresis generated by the antagonistic SMA wires. The maximum overshoot was observed to be $13.2 \%$ in $X$-axis and $21 \%$ for $Z$-axis, as well as, the maximum steady state error was $0.0123 \mathrm{~m}$ for $X$-axis and $0.0057 \mathrm{~m}$ for $Z$-axis, which correspond to the $0.7 \%$ and $1.6 \%$ respectively. The average settling time was 6 seconds.

Future work will be orientated to construct and to test experimentally the presented design. In addition, the proposed SMA based robotic arm will be attached to a small quadcopter, which is assembled as flying manipulator. Furthermore an ON/OFF control will be developed to open and close the gripper. The gripper will be actuated by a third SMA wire biased with a compression spring, avoiding the use of electric motor or any other kind of actuators besides SMA wires.

\section{REFERENCES}

[1] J. Colorado, A. Barrientos, C. R., 2011. "Músculos inteligentes en robots biológicamente inspirados: Modelado, control y actuación". Revista Iberoamericana de Automática e Informática Industrial RIAI, 8(4), pp. 385 396.

[2] Nematzadeh, F., and Sadrnezhaad, S., 2012. "Effects of material properties on mechanical performance of nitinol stent designed for femoral artery: Finite element analysis". Scientia Iranica, 19(6), pp. $1564-1571$.

[3] Pandis, N., and Bourauel, C. P., 2010. "Nickel-titanium (niti) arch wires: the clinical significance of super elas- 
ticity". In Seminars in Orthodontics, Vol. 16, Elsevier, pp. 249-257.

[4] Sreekumar, M., Nagarajan, T., and Singaperumal, M., 2009. "Application of trained niti sma actuators in a spatial compliant mechanism: Experimental investigations". $M a$ terials \& Design, 30(8), pp. 3020-3029.

[5] Liu, S. H., Huang, T.-S., and Yen, J. Y., 2008. "Sensor fusion in a sma-based hexapod bio-mimetic robot". In 2008 IEEE Workshop on Advanced robotics and Its Social Impacts, pp. 1-6.

[6] Feng, Y., Rabbath, C. A., Hong, H., Su, C. Y., and Lin, W., 2010. "Inverse hysteresis control for shape memory alloy micro-actuators based flap positioning system". Proceedings of the IEEE Conference on Decision and Control, pp. 3662-3667.

[7] Son, H.-M., Kim, M. Y., and Lee, Y.-J., 2009. "Tunablefocus liquid lens system controlled by antagonistic winding-type SMA actuator". Optics Express, 17(16), jul, p. 14339.

[8] Williams, E. A., Shaw, G., and Elahinia, M., 2010. "Control of an automotive shape memory alloy mirror actuator". Mechatronics, 20(5), pp. 527-534.

[9] Quintanar-Guzmán, S., Reyes-Reyes, J., and ArellanoSánchez, M. d. c., 2014. "Modelado y control de un sistema electrotérmico-mecánico móvil basado en alambres musculares". In XVI Congreso Latinoamericano de Control Automtico, CLCA 2014, Asociación de México de Control Automático, pp. 834-839.

[10] Kannan, S., Giraud-Audine, C., and Patoor, E., 2013. “Application of laguerre based adaptive predictive control to shape memory alloy (sma) actuator". $\{$ ISA $\}$ Transactions, 52(4), pp. $469-479$.

[11] Elahinia, M. H., and Ashrafiuon, H., 2002. "Nonlinear Control of a Shape Memory Alloy Actuated Manipulator". Journal of Vibration and Acoustics, 124(4), p. 566.

[12] Guo, Z., Pan, Y., Wee, L. B., and Yu, H., 2015. "Design and control of a novel compliant differential shape memory alloy actuator". Sensors and Actuators A: Physical, 225, apr, pp. 71-80.

[13] Kannan, S., Giraud-Audine, C., and Patoor, E., 2010. "Laguerre model based adaptive control of antagonistic shape memory alloy (sma) actuator". In SPIE Smart Structures and Materials+ Nondestructive Evaluation and Health Monitoring, International Society for Optics and Photonics, pp. 764307-764307.

[14] Kannan, S., Giraud-Audine, C., and Patoor, E., 2009. "Control of shape memory alloy (sma) actuator using seriesparallel model reference adaptive control (mrac)". In ASME 2009 Conference on Smart Materials, Adaptive Structures and Intelligent Systems, American Society of Mechanical Engineers, pp. 441-450.

[15] Kannan, S., 2011. "Modélisation et commande daction- neurs à alliage à mémoire de forme”. PhD thesis, l'École Nationale Supérieure d'Arts et Métiers.

[16] Hulea, M., and Caruntu, C. F., 2014. "Spiking neural network for controlling the artificial muscles of a humanoid robotic arm". pp. 163-168.

[17] Khodayari, A., Talari, M., and Kheirikhah, M. M., 2011. "Fuzzy PID controller design for artificial finger based SMA actuators". IEEE International Conference on Fuzzy Systems, pp. 727-732.

[18] Ko, J., Jun, M. B., Gilardi, G., Haslam, E., and Park, E. J., 2011. "Fuzzy PWM-PID control of cocontracting antagonistic shape memory alloy muscle pairs in an artificial finger". Mechatronics, 21(7), pp. 1190-1202.

[19] Bellicoso, C. D., Buonocore, L. R., Lippiello, V., and Siciliano, B., 2015. "Design, modeling and control of a 5DoF light-weight robot arm for aerial manipulation". 2015 23rd Mediterranean Conference on Control and Automation, MED 2015 - Conference Proceedings, pp. 853-858.

[20] Liang, C., and Rogers, C. A., 1990. "One-dimensional thermomechanical constitutive relations for shape memory materials". Journal of intelligent material systems and structures, 1(2), pp. 207-234.

[21] Slotine, J., and Li, W., 1991. Applied Nonlinear Control. Prentice Hall.

[22] Siciliano, B., Sciavicco, L., Villani, L., and Oriolo, G., 2010. Robotics: modelling, planning and control. Springer Science \& Business Media.

[23] Flexinol actuator wire technical and design data. http: // www.dynalloy.com/tech_data_wire.php. Accessed: 2016-04-26.

[24] Technical characteristics of flexinol. http://www. dynalloy.com/pdfs/TCF1140.pdf. Accessed: 2016-04-26. 